\title{
Spatial homogeneity of optically switched semiconductor photonic crystals and of bulk semiconductors
}

\author{
Tijmen G. Euser ${ }^{\mathrm{a})}$ and Willem L. Vos \\ Complex Photonic Systems (COPS), Department of Science and Technology and MESA ${ }^{+}$Research Institute, \\ University of Twente, P.O. Box 217, 7500 AE Enschede, The Netherlands
}

(Received 10 September 2004; accepted 10 November 2004; published online 20 January 2005)

\begin{abstract}
In this paper we discuss free carrier generation by pulsed laser fields as a mechanism to switch the optical properties of semiconductor photonic crystals and bulk semiconductors on an ultrafast time scale. Requirements are set for the switching magnitude, the time-scale, the induced absorption, as well as the spatial homogeneity, in particular for silicon at $\lambda=1550 \mathrm{~nm}$. Using a nonlinear absorption model, we calculate carrier depth profiles and define a homogeneity length $l_{\text {hom }}$. Homogeneity length contours are visualized in a plane spanned by the linear and two-photon absorption coefficients. Such a generalized homogeneity plot allows us to find optimum switching conditions at pump frequencies near $\nu / c=5000 \mathrm{~cm}^{-1}(\lambda=2000 \mathrm{~nm})$. We discuss the effect of scattering in photonic crystals on the homogeneity. We experimentally demonstrate a $10 \%$ refractive index switch in bulk silicon within $230 \mathrm{fs}$ with a lateral homogeneity of more than $30 \mu \mathrm{m}$. Our results are relevant for switching of modulators in the absence of photonic crystals. (C) 2005 American Institute of Physics. [DOI: 10.1063/1.1846949]
\end{abstract}

\section{INTRODUCTION}

There is a fast growing interest in photonic crystals; composite materials whose refractive index varies periodically on length scales that match the wavelength of light. ${ }^{1}$ The optical properties of photonic materials are determined by the spatially varying refractive index, analogous to the periodic potential for an electron in a crystal. Large spatial variations of the refractive index cause a strong interaction between light and the composite structure. Bragg diffraction causes the photonic dispersion to organize into bands, much like the energy levels of electrons in semiconductors. A major goal of the field is the realization of three-dimensional (3-D) structures that possess a photonic band gap. ${ }^{2,3}$ At frequencies inside the band gap, the optical density of states vanishes. This should completely inhibit spontaneous emission of sources inside the photonic crystal. ${ }^{2}$ Indeed, strong modifications of the spontaneous emission lifetime of quantum dots have recently been demonstrated in photonic crystals. ${ }^{4}$ In the presence of weak controlled disorder, the Anderson localization of light is also predicted. ${ }^{3}$ In this case, a photon may be trapped at a point defect that serves as a cavity with a high quality factor. ${ }^{5}$

In the examples above, the photonic crystals themselves do not change in time. Switching experiments, in which the properties of photonic crystals are modified on an ultrafast time scale, allow many interesting new opportunities. Switching 3-D photonic crystals is particularly interesting, as it provides the dynamic control over the density of states inside the crystal as well as a change in Bragg reflections. ${ }^{6}$ Ultrafast control of the density of states should allow the switching of the spontaneous emission of light sources inside a crystal, and capturing and releasing light in cavities inside the crystal. In the absence of photonic crystals, ultrafast

${ }^{a)}$ Electronic mail: T.G.Euser@utwente.nl switching of bulk semiconductors finds applications in high speed optical modulators, ${ }^{7}$ waveguides, ${ }^{8}$ and multilayer stacks. ${ }^{9}$ Last but not least, ultrafast control of photonic crystals is important for controlling the propagation of light, such as in switched macroporous silicon, ${ }^{10}$ or 2-D crystal slabs. ${ }^{11}$

In optical switching experiments, four important requirements have to be met. ${ }^{6}$ First of all, the magnitude of the induced change in the real part of the refractive index $n^{\prime}$ must be large enough to obtain the desired effect. A relative change in $n^{\prime}$ of $5 \%$ is required to induce a major change in the density of states. Such large changes can be induced by free carrier generation ${ }^{12}$ but are not achievable with Kerr switching. The second requirement, which is important for applications, is the minimum time scale $\Delta t$ over which the switch occurs. In experiments where light pulses are trapped inside photonic crystals, a switching time scale on the order of a few hundred femtoseconds is necessary. The third requirement is that the absorption of probe light, gauged by $n^{\prime \prime}$, should be small in the switched sample. Excited carriers in a semiconductor bring about inevitable absorption (related by a Kramers-Kronig relation to the change in $n^{\prime}$ ), that should remain within limits by a limited carrier density. The fourth requirement concerns the spatial homogeneity of the change $\Delta n^{\prime}$ in a sample. Homogeneity is particularly important in switching the density of states in 3-D photonic crystals. A large gradient in $\Delta n^{\prime}$ in the crystal results in a highly chirped switched sample, which can no longer be considered a photonic crystal. All of these requirements also pertain to other applications of switched semiconductors, such as in waveguiding, ${ }^{7}$ albeit much relaxed. Therefore, we expect the results from the present study also to be relevant for applications outside photonic crystals.

Switching experiments in photonic crystals were pioneered by Leonard et al. ${ }^{10}$ Optical free carrier generation was used to change the refractive index of the backbone of a 2-D 


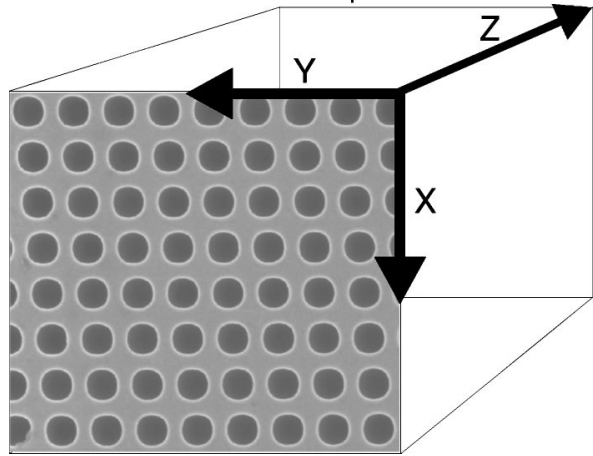

FIG. 1. Schematic image of a sample: The $z$ axis is defined to be pointing into the sample; the $x$ and $y$ directions are the lateral directions. In the figure, the front face of the sample displays a SEM image of a dry etched Si 2-D photonic crystal with a slightly rhomboid symmetry (lattice angle $85^{\circ}$ ). The lattice parameter $a$ is equal to $750 \mathrm{~nm}$. SEM courtesy of L. A. Woldering.

silicon photonic crystal. The carrier density generated in their experiment was sufficiently high to induce a shift of a Bragg stopgap on ultrafast time scales. Nevertheless, the experiments revealed a serious limitation: the absorption of the pump beam limits the volume of switched material and leads to a spatial inhomogeneity in the degree of switching. Leonard et al. deduced that only a layer of three unit cells near the sample surface was switched. ${ }^{10}$ Similar inhomogeneity is probably also playing a role in recent studies of silicon infiltrated opaline crystals, where a disappearance of the Bragg peak was observed in the absorption range. ${ }^{13}$ Therefore, the use of two-photon absorption was proposed as a way to increase the penetration depth of pump light into the sample and improve the switching homogeneity. ${ }^{6}$

In this paper, we investigate the spatial homogeneity of optically generated free carrier plasmas in semiconductors. We discuss a nonlinear absorption model that takes into account both linear and two-photon absorption processes. From this model, we derive optimum pumping conditions and we define a homogeneity length scale to obtain homogeneous switching conditions. In particular, we trace constant homogeneity lengths in a general parameter diagram that pertains to any semiconductor. We discuss the role of disorderinduced diffusion of the pump beam in photonic crystals. Finally, we experimentally demonstrate that sufficient ultrafast refractive index changes can be obtained with sufficient lateral homogeneity.

We concentrate on silicon, not only because of its wide technological use, but also because it allows greater homogeneity than GaAs that was discussed in Ref. 6. Examples are given for light in the telecom band at $\nu_{\text {tele }} / c$ $=6450 \mathrm{~cm}^{-1}(\lambda=1550 \mathrm{~nm})$, but can easily be generalized to other frequencies. We set a minimum volume of five unit cells cubed in which the change $\Delta n^{\prime}$ must remain within $10 \%$ of its maximum value. This homogeneity requirement holds for both the lateral directions $x$ and $y$, as well as for the $z$ direction defined in Fig. 1. Even in such a small crystal volume, the DOS already shows a significant decrease for frequencies that lie in the band gap. ${ }^{14}$ The typical unit cell size of silicon inverse opal photonic crystals with a bandgap

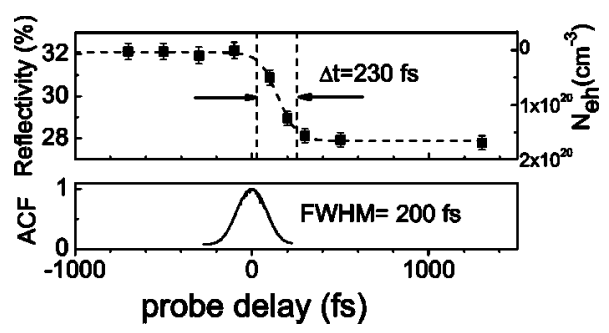

FIG. 2. Time-resolved reflectivity measurement on bulk $\mathrm{Si}$, pumped at $\nu_{\text {pump }} / c=12500 \mathrm{~cm}^{-1}$, pulse energy $E_{\text {pump }}=2 \mu \mathrm{J}$, pulse duration $\tau_{\text {pump }}$ $=120 \mathrm{fs}, r_{\text {pump }}=70 \mu \mathrm{m}$ and peak intensity $115 \mathrm{GW} / \mathrm{cm}^{2}$ (upper panel). The reflectivity of a probe with $\nu_{\text {probe }} / c=7692 \mathrm{~cm}^{-1}, r_{\text {probe }}=20 \mu \mathrm{m}$ and $\tau_{\text {probe }}$ $=120 \mathrm{fs}$ decreases from $32 \%$ to $28 \%$, corresponding to a calculated carrier density $N_{\mathrm{eh}}=1.6 \times 10^{20} \mathrm{~cm}^{-3}$ at the surface of the sample (see the right-hand scale). The time in which the relative reflectivity change increases from $10 \%$ to $90 \%$ is $230 \mathrm{fs}$. The lower panel shows the intensity autocorrelation function (ACF) of the pump pulses. The full width at half-maximum (FWHM) of 200 fs corresponds to a Gaussian pulse width of 140 fs FWHM.

near $\nu_{\text {tele }}$ is $a=1.2 \mu \mathrm{m}$, therefore the homogeneously switched area must extend at least $6 \mu \mathrm{m}$ in all three dimensions.

\section{FREE CARRIER GENERATION}

In optical free carrier generation, a pump pulse is absorbed by a semiconductor sample, creating a free carrier plasma with electron-hole density $N_{\text {eh }}$. The generated carrier plasma changes the dielectric function $\epsilon(\omega)$ of the sample by an amount $\Delta \epsilon_{\mathrm{eh}}(\omega)$. In the case of silicon, the Drude model gives on excellent description of the resulting $\epsilon(\omega)$ for densities below $10^{22} \mathrm{~cm}^{-3}$ (see Ref. 12):

$$
\epsilon(\omega)=\epsilon_{B}(\omega)+\Delta \epsilon_{\mathrm{eh}}(\omega)=\epsilon_{B}-\left(\frac{\omega_{p}}{\omega}\right)^{2} \frac{1}{1+i \frac{1}{\omega \tau_{\mathrm{D}}}},
$$

where $\epsilon_{B}$ is the bulk dielectric constant, $\omega(=2 \pi \nu)$ is the frequency of the probe light, $m_{e}$ the electron mass, $m_{\mathrm{opt}}^{*}$ is the optical effective mass of the carriers, $\tau_{\mathrm{D}}$ the Drude damping time, ${ }^{15}$ and $\omega_{p}=\sqrt{\left(N_{\mathrm{eh}} e^{2}\right) /\left(\epsilon_{0} m_{\mathrm{opt}}{ }^{*} m_{e}\right)}$ the plasma frequency. Under the condition that $1 /\left(\omega \tau_{\mathrm{D}}\right) \ll 1$, which is valid for silicon at $\omega_{\text {tele }}$, we can derive the following simplified expression for the refractive index from Eq. (1):

$$
\begin{aligned}
n^{\prime}+i n^{\prime \prime} & =\sqrt{\epsilon(\omega)} \\
& =\sqrt{\epsilon_{B}-\left(\frac{\omega_{p}}{\omega}\right)^{2}}+i \frac{\omega_{p}^{2}}{2 \omega^{3} \tau_{\mathrm{D}} \sqrt{\epsilon_{B}-\left(\frac{\omega_{p}}{\omega}\right)^{2}}} .
\end{aligned}
$$

For silicon with carrier densities below $10^{20} \mathrm{~cm}^{-3}$, the refractive index is linear with the carrier density within $0.2 \%$ :

$$
n^{\prime}=\sqrt{\epsilon_{B}}-\frac{e^{2}}{2 \sqrt{\epsilon_{B}} \epsilon_{0} m_{\mathrm{opt}}^{*} m_{e} \omega^{2}} N_{\mathrm{eh}} .
$$

Thus, the induced change in the refractive index $n^{\prime}$ [via $\epsilon(\omega)]$ is completely determined by the optically induced carrier density.

An example of a carrier-induced change of the refractive index in bulk silicon is given in Fig. 2. In this experiment, a 
powerful ultrashort pump pulse with frequency $\nu_{\text {pump }} / c$ $=12500 \mathrm{~cm}^{-1}$ and a pulse width of $140 \mathrm{fs}$ was focused to a spot with radius $r_{\text {pump }}=70 \mu \mathrm{m}$, resulting in a peak intensity at the sample interface of $I_{0}=115 \mathrm{GW} \mathrm{cm}^{-2}$. The reflectivity of a weaker probe pulse with frequency $\nu_{\text {probe }} / c$ $=7692 \mathrm{~cm}^{-1}$, a pulse width of $140 \mathrm{fs}$, and a smaller spot radius of $r_{\text {probe }}=20 \mu \mathrm{m}$ was measured in the center of the pumped spot at different time delays with respect to the pump pulse. The scan in the upper part of Fig. 2 shows that the reflectivity of the sample changes from $32 \%$ to $28 \%$. The switching time is taken as the time in which the relative reflectivity change increases from $10 \%$ to $90 \%$, corresponding to a reflectivity of $31.6 \%$ and $28.4 \%$, respectively. From Fig. 2 we obtain a switching time of $230 \mathrm{fs}$, confirming an ultrafast change in $n^{\prime}$.

From Fresnel's formula we find the refractive index change to be more than $10 \%$, corresponding to a large generated carrier density of $1.6 \times 10^{20} \mathrm{~cm}^{-3}$. This is about twice the carrier density that is needed to obtain a change in $n^{\prime}$ of $5 \%$ for probe light at $\omega_{\text {tele. }}$. We have shown that optical carrier generation can be used to change $n^{\prime}$ by a large amount on a subpicosecond time scale, meeting the first two of the four main density of states switching requirements.

For applications such as optical modulators and waveguides, much smaller changes in $n^{\prime}$ are already sufficient, typically $\Delta n^{\prime}=10^{-4}$; see Ref. 7 . This corresponds to a carrier density of $10^{16} \mathrm{~cm}^{-3}$. For such low carrier densities, the required pump pulse energy at $\nu_{\text {pump }} / c=12500 \mathrm{~cm}^{-1}$ is on the order of several $\mathrm{nJ}$, allowing the use of diode lasers with repetition rates exceeding $1 \mathrm{GHz}$ as a pump source. Thus, it seems that carrier-induced optical switching may have much broader applications beyond photonic crystals.

Next, we will discuss the induced absorption in switched semiconductors, which is the third density of states switching requirement. The carrier absorption length $l_{\text {ca }}$ of the excited carrier plasma is equal to

$$
l_{\mathrm{ca}}=\frac{1}{2 k_{0} n^{\prime \prime}(\omega)},
$$

where $k_{0}=\omega n^{\prime}(\omega) / c$. After inserting the expressions for $n^{\prime}(\omega)$ and $n^{\prime \prime}(\omega)$ from Eq. (2), we get

$$
l_{\mathrm{ca}}=\left(\frac{\omega}{\omega_{p}}\right)^{2} \tau_{\mathrm{D}} c .
$$

We immediately see that the absorption length is inversely proportional to the plasma frequency $\omega_{p}$ squared and thus inversely proportional to the carrier density. In silicon, the carrier absorption length of probe light at $\omega_{\text {tele }}$ is $l_{\text {ca }}$ $=22 \mu \mathrm{m}$ for a carrier density of $10^{20} \mathrm{~cm}^{-3}$. In our analysis of 3-D photonic crystals we assume the crystals to be inverse opals with a typical filling silicon filling fraction of $\Phi$ $=25 \%$. The carrier absorption length in such crystals will then be approximately four times larger than the bulk carrier absorption length, or $l_{\mathrm{ca}}=88 \mu \mathrm{m}$. This result shows that in our analysis, where the carrier density is less than $10^{20} \mathrm{~cm}^{-3}$, the carrier induced absorption for $\omega_{\text {tele }}$ remains very small. We have now shown that the first three requirements for successful switching experiments can be met for silicon at

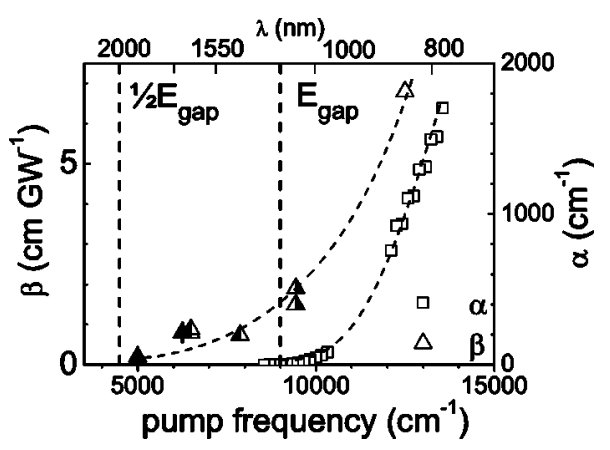

FIG. 3. Absorption coefficients $\alpha$ and $\beta$ of Si versus pump frequency. The dashed vertical lines correspond to photon energies of $E_{\text {gap }} / 2$ and $E_{\text {gap }}$. The open squares indicate linear absorption coefficients taken from Ref. 16 (right-hand scale). The solid triangles indicate the two-photon absorption coefficients that we have determined by $z$-scan measurements (left-hand scale). The left-filled triangles indicate the values from Ref. 17, the rightfilled triangles are data from Ref. 18, and the open triangle data from Ref. 19.

telecom frequencies. The remainder of this paper will discuss the fourth requirement: the spatial switching homogeneity.

\section{OPTICAL PROPERTIES OF SILICON}

To analyze the switching homogeneity in the $z$ direction, we briefly consider how pump light is absorbed in semiconductors. At low pump intensities $I$, the absorption of light in semiconductors scales with the intensity: $\alpha I$, where the absorption coefficient $\alpha$ tends to zero for photon energies $\hbar \omega$ below the electronic bandgap energy $E_{\text {gap }}$; see Fig. 3 for silicon. At high pumping intensities, nonlinear two-photon absorption starts to play an important role. For two-photon absorption, the absorption is proportional to $\beta I^{2}$, where $\beta$ is the two-photon absorption coefficient. This coefficient is expected to vanish for $\hbar \omega>E_{\text {gap }} / 2$. Figure 3 displays the frequency dependence of the linear and two-photon absorption coefficients of silicon. The data was obtained from our measurements and from Refs. 16-19: With our laser system, ${ }^{20}$ $z$-scan measurements ${ }^{21}$ were performed to obtain the twophoton absorption coefficient $\beta$ at two additional wavelengths. For bulk silicon at $\nu_{\text {pump }} / c=5000 \mathrm{~cm}^{-1}, \beta$ was measured to be $0.20 \pm 0.05 \mathrm{~cm} \mathrm{GW}^{-1}$. At $\nu_{\text {pump }} / c=6250 \mathrm{~cm}^{-1}$, we obtained $\beta=0.80 \pm 0.1 \mathrm{~cm} \mathrm{GW}^{-1}$. The latter value is in excellent correspondence with the value $\beta=0.88 \pm 0.13 \mathrm{~cm} \mathrm{GW}^{-1}$ at $\nu_{\text {pump }} / c=6494 \mathrm{~cm}^{-1}$ from Ref. 17 . Our measurements confirm that $\beta$ tends to zero for photon energies approaching $E_{\text {gap }} / 2$. Both the linear and the nonlinear absorption coefficient can thus be controlled by varying the pump frequency.

\section{HOMOGENEITY OF SWITCHED SEMICONDUCTORS}

\section{A. Homogeneity in the $z$ direction}

We now present a model that calculates the carrier density depth profile $N_{\text {eh }}(z)$ caused by the absorption of pump light. The absorbed intensity is described by the nonlinear differential equation 


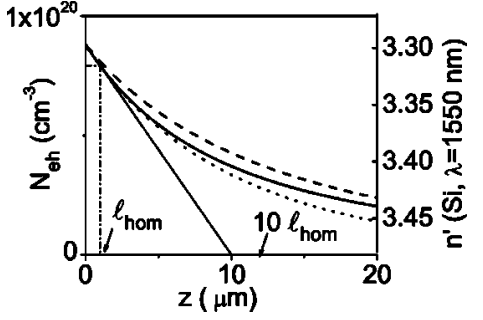

FIG. 4. Calculated carrier density depth profile $N_{\mathrm{eh}}(z)$ for constant $\beta$ $=2 \mathrm{~cm} \mathrm{GW}^{-1}$ and three different linear absorption coefficients: $\alpha=0$ (solid curve), $\alpha=400 \mathrm{~cm}^{-1}$ (dashed curve) and $\alpha=800 \mathrm{~cm}^{-1}$ (dotted curve). With $N_{\text {eh }}$ at the interface kept constant at $0.9 \times 10^{20} \mathrm{~cm}^{-3}$, the necessary pump intensity $I_{0}$ is calculated, assuming that $\tau_{\text {pump }}=120 \mathrm{fs}$ and $r_{\text {pump }}=75 \mu \mathrm{m}$. The corresponding carrier density depth profile was obtained with Eq. (7). The homogeneity length $l_{\text {hom }}$ is shown for $\alpha=0$. The right-hand scale shows the resulting refractive index $n^{\prime}$ for $\nu / c=6450 \mathrm{~cm}^{-1}$ light in $\mathrm{Si}$.

$$
\frac{d I(z)}{d z}=-\left[\alpha I(z)+\beta I^{2}(z)\right]
$$

which we have solved by implicit integration. The resulting expression describing the intensity depth profile is

$$
I(z)=\frac{I_{0} e^{-\alpha z}}{1+\left(\beta I_{0} / \alpha\right)\left(1-e^{-\alpha z}\right)},
$$

where $I_{0}$ is the intensity at the interface. The resulting carrier density profile $N_{\mathrm{eh}}(z)$ is related to the intensity depth profile $I(z)$ as

$$
N_{\text {eh }}(z)=\frac{I(z) \tau_{\text {pump }}}{\hbar \omega_{\text {pump }}}\left[\alpha+\frac{1}{2} \beta I(z)\right],
$$

where $\tau_{\text {pump }}$ is the pump pulse duration. The factor $1 / 2$ for two-photon absorption indicates that two photons must be absorbed to generate one electron-hole pair. The substitution of Eq. (7) into Eq. (8) allows us to calculate the carrier density profile for any given combination of $\alpha, \beta$, and $I_{0}$.

We now investigate the relation between the absorption coefficients and pumping homogeneity. To quantify the homogeneity of a switched sample, we first define the homogeneity length $l_{\text {hom }}$ within which the carrier density remains within $10 \%$ of its surface value:

$$
l_{\text {hom }} \equiv 0.1 \times\left[\frac{1}{N_{\mathrm{eh}}(z)} \frac{d N_{\mathrm{eh}}(z)}{d z}\right]_{z=0} .
$$

Because the homogeneity length is directly related to the maximum gradient in the carrier depth profile, it is a helpful parameter in quantifying the homogeneity of switched semiconductors. For applications where switching homogeneity is important, the homogeneity length should be much larger than the size of the switched sample. To illustrate the homogeneity length, three carrier density depth profiles are shown in Fig. 4 for which $\beta$ is kept constant at $2 \mathrm{~cm} \mathrm{GW}^{-1}$, and $\alpha$ is varied from zero (dotted curve) to $400 \mathrm{~cm}^{-1}$ (dashed curve) to $800 \mathrm{~cm}^{-1}$ (solid curve). For each case, $I_{0}$ is chosen such that the carrier density reaches $0.9 \times 10^{20} \mathrm{~cm}^{-3}$ at the sample interface, corresponding to a $5 \%$ change in $n^{\prime}$ at $\nu_{\text {tele }}$ in silicon. On the right $y$ axis the corresponding real part of the refractive index $n^{\prime}$ for silicon at $\nu_{\text {tele }}$ is shown. The pump frequency was assumed to be $5000 \mathrm{~cm}^{-1}$. A closer look at the

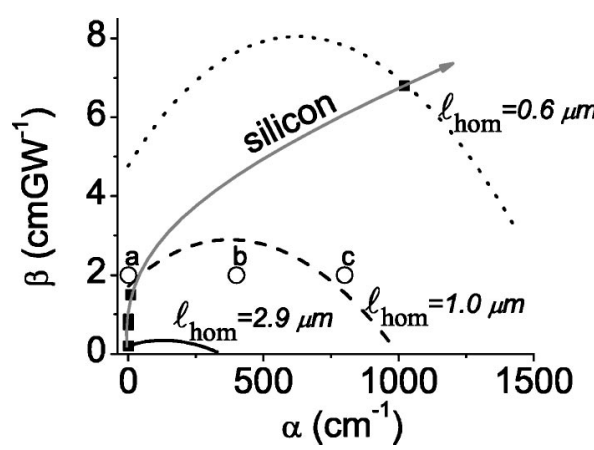

FIG. 5. Homogeneity plot. Contours of constant $l_{\text {hom }}$ are plotted in the $(\alpha, \beta)$ plane, defining regions in which homogeneous switching can be achieved. The generated carrier density is kept constant at $N_{\text {eh }}(0)=0.9 \times 10^{20} \mathrm{~cm}^{-3}$, enough for a $5 \%$ change in $n^{\prime}$. The solid curve corresponds to $l_{\text {hom }}$ $=2.9 \mu \mathrm{m}$, the dashed curve to $l_{\text {hom }}=1.0 \mu \mathrm{m}$, and the dotted curve to $l_{\text {hom }}$ $=0.6 \mu \mathrm{m}$ for bulk silicon. The closed squares connected by the dotted arrow are linear and two-photon coefficients for Si obtained from Fig. 2. The open circles a, b, and c correspond to the depth profiles $N_{\text {eh }}(z)$ plotted in Fig. 4. Pumping parameters: $\tau_{\text {pump }}=120 \mathrm{fs}, \quad r_{\text {pump }}=75 \mu \mathrm{m} ; \quad$ and $\nu_{\text {pump }} / c$ $=5000 \mathrm{~cm}^{-1}$.

three depth profiles in Fig. 4 shows that for $\alpha=0 \mathrm{~cm}^{-1}$, the homogeneity length is $1.0 \mu \mathrm{m}$. As $\alpha$ increases to $400 \mathrm{~cm}^{-1}$, the homogeneity length increases to $1.2 \mu \mathrm{m}$. As $\alpha$ increases further to $800 \mathrm{~cm}^{-1}$, the homogeneity length decreases again to $1.0 \mu \mathrm{m}$. The surprising occurrence of a maximum in the homogeneity length can be explained with the aid of Eq. (8). For small $\alpha\left(\alpha \ll I_{0} \beta\right)$, the absorption is dominated by twophoton absorption. If $\alpha$ increases, the pump intensity $I_{0}$ needed to obtain the surface carrier density decreases, reducing the slope of the intensity profile at the interface, determined by the exponent $-\left(\alpha+I_{0} \beta\right)$. This leads to an increase in $l_{\text {hom }}$. If $\alpha$ increases further to the regime where the absorption is dominated by linear absorption $\left(\alpha \gg I_{0} \beta\right)$, any further increase in $\alpha$ will result in a decrease of the homogeneity length. In the region between the two extremes, the homogeneity length apparently attains a maximum value. This means that simply choosing two-photon (or perhaps even higherphoton) absorption over linear absorption is not always sufficient to ensure an optimal homogeneity.

We make a homogeneity plot to obtain further insight in the influence of $\alpha$ and $\beta$ on the homogeneity. First we choose a fixed electron density at the interface $N_{\text {en }}(0)=0.9$ $\times 10^{20} \mathrm{~cm}^{-3}$. The corresponding homogeneity length contours are then deduced from our absorption model, and visualized in a plane spanned by linear and two-photon absorption coefficients; see Fig. 5. The absorption coefficients for silicon at various frequencies taken from Fig. 3 are also plotted in the plane in Fig. 5. The three depth profiles shown in Fig. 4 correspond to positions a, b, and c in Fig. 5. To obtain a certain minimum homogeneity, the absorption coefficients must remain below the curve corresponding to the particular minimum homogeneity. This graph thus allows us to directly obtain the homogeneity length that can be obtained for a semiconductor at a certain frequency. The homogeneity plot demonstrates how pumping homogeneity can be maximized by choosing the appropriate pump frequency. Generally, smaller absorption coefficients lead to an increased homogeneity. This increase comes at the price of a higher necessary 


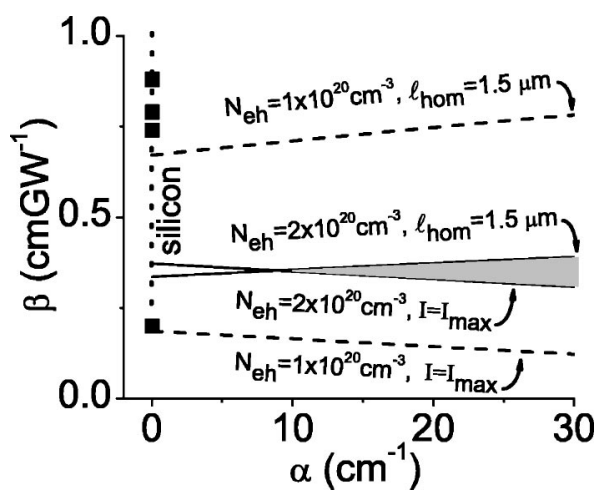

FIG. 6. Carrier density contours for a particular bulk homogeneity chosen as $l_{\text {hom }}=1.5 \mu \mathrm{m}$. The contours correspond to two different values for $N_{\text {eh }}(0)$ : the dashed lines to $N_{\mathrm{eh}}(0)=1 \times 10^{20} \mathrm{~cm}^{-3}$, and the solid lines to $N_{\mathrm{eh}}(0)=2$ $\times 10^{20} \mathrm{~cm}^{-3}$. The upper one of each pair of curves corresponds to $l_{\text {hom }}$ $=1.5 \mu \mathrm{m}$. The lower curve of each pair indicates the minimum absorption coefficients for which the given $N_{\mathrm{eh}}(0)$ can be obtained without exceeding the maximum intensity $I_{\max }=1 \mathrm{TW} \mathrm{cm}^{-2}$. The closed squares connected by the dotted curve are the $\alpha$ and $\beta$ coefficients for Si obtained from Fig. 3. Pumping parameters: $\tau_{\text {pump }}=120 \mathrm{fs}, \quad r_{\text {pump }}=75 \mu \mathrm{m}, \quad$ and $\nu_{\text {pump }} / c$ $=5000 \mathrm{~cm}^{-1}$.

pump intensity $I_{0}$. From Fig. 5 we conclude that the most homogeneous switch for silicon can be achieved for $\nu_{\text {pump }} / c=5000 \mathrm{~cm}^{-1}$. For this pump frequency, the homogeneity length is $2.9 \mu \mathrm{m}$. For comparison, if $\nu_{\text {pump }} / c$ is equal to $12500 \mathrm{~cm}^{-1}$, the homogeneity length is only $0.6 \mu \mathrm{m}$. The necessary pump intensity remains below the maximum available pump energy of our laser system $I_{\max }$. Our generalized homogeneity plot is valid for all materials and allows us to find optimum switching conditions. The choice for pumping frequencies that are low in the two-photon absorption regime drastically increases the pumping homogeneity.

We now make a plot that shows surface carrier density contours $\left[N_{\mathrm{eh}}(0)\right]$ corresponding to a particular fixed homogeneity length. From such a plot the maximum homogeneous change in refractive index can be derived. We choose a fixed homogeneity length of $1.5 \mu \mathrm{m}$, since inside a typical photonic crystal with a $25 \%$ filling fraction, the homogeneity length will be four times larger $(6 \mu \mathrm{m})$, thus fulfilling our homogeneity requirement. Figure 6 displays contours for two different carrier densities $N_{\mathrm{eh}}=1 \times 10^{20} \mathrm{~cm}^{-3}$ and 2 $\times 10^{20} \mathrm{~cm}^{-3}$.

In Fig. 6, the upper curve is the homogeneity contour, and the lower one of each pair of curves indicates the minimum absorption coefficients for which carrier density can be generated given the maximum available intensity $I_{\max }$ $=1 \mathrm{TW} \mathrm{cm}{ }^{-2} \cdot{ }^{20}$ For feasible switching experiments, the absorption coefficients must be in the area to the right of the intersection of the two curves. As the carrier density increases, the constant $l_{\text {hom }}$ line moves toward lower absorption coefficients, while the $I_{\max }$ line moves toward higher values. As an example, for a carrier density of 2 $\times 10^{20} \mathrm{~cm}^{-3}$, homogeneous switching can only be achieved for absorption coefficients within the shaded area in Fig. 6. It is seen that this area does not overlap with the trajectory of silicon parameters. Therefore, this carrier density is not achievable, given $l_{\text {hom }}=1.5 \mu \mathrm{m}$. With decreasing carrier density, the range between the curves will overlap the silicon parameter trajectory at some point. Such an intersection determines the upper limit to the carrier density (given $l_{\text {hom }}$ $=1.5 \mu \mathrm{m})$ as well as the pump frequency that pertains to the relevant $(\alpha, \beta)$ point. For silicon, this intersection is calculated to occur at $N_{\mathrm{eh}}=1.9 \times 10^{20} \mathrm{~cm}^{-3}$. From Eq. (3) we obtain the corresponding maximum homogeneous change in the refractive index at $\nu_{\text {tele }}$ to be $11 \%$.

The condition of a maximum intensity $I_{\max }$ can be relaxed by choosing a smaller pump spot radius $r_{\text {pump }}$. The assumed pump radius of $75 \mu \mathrm{m}$ provides a lateral homogeneity that is large compared to the homogeneity requirement; therefore we could choose a smaller $r_{\text {pump }}$ while maintaining sufficient lateral homogeneity. A higher pump intensity would allow homogeneous switching experiments at even lower absorption coefficients, allowing larger changes of the refractive index. However, we must keep in mind that the carrier absorption length is inversely proportional to the induced carrier density; see Eq. (5). For a refractive index change of $11 \%$, we predict the carrier absorption length inside a photonic crystal to drop to $l_{\mathrm{ca}}=38 \mu \mathrm{m}$, which may be sufficient to meet our third requirement for switching experiments in photonic crystals. At higher carrier densities, however, the carrier absorption length will become too small to meet this requirement.

A similar analysis can be done for other semiconductor materials. The carrier density needed for a 5\% change in $n^{\prime}$ will depend on material properties such as the optical effective mass of the carriers and the Drude damping time $\tau_{\mathrm{D}}$ [see Eq. (1)]. We briefly discuss switching of GaAs at a frequency $\nu / c=9430 \mathrm{~cm}^{-1}$ proposed in Ref. 6. For GaAs the optical effective mass $m_{\mathrm{opt}}^{*}=0.06$ is 2.5 times smaller than for silicon. $^{22}$ Therefore, the carrier density in GaAs is 2.5 times lower than what is required for the same refractive index change in silicon. We find that the area increases in which the homogeneity requirement can be met. However, the twophoton absorption coefficient for GaAs at $\nu / c=9430 \mathrm{~cm}^{-1}$ is $26 \mathrm{~cm} \mathrm{GW}^{-1}$, far above the homogeneous switching area. ${ }^{22}$ This immediately shows, that GaAs at this pump frequency cannot be used in homogeneous switching experiments. From this brief analysis we learn that the pumping frequencies in GaAs should be reduced, to where the two-photon absorption coefficient is a much lower value.

\section{B. Lateral homogeneity}

We now consider the homogeneity in the lateral $(x, y)$ directions. We have measured the relative change in probe reflectivity from a bulk silicon sample pumped in the twophoton absorption regime at $\nu_{\text {pump }} / c=6250 \mathrm{~cm}^{-1}$; see Fig. 7 . For this frequency, $\beta=0.8 \pm 0.1 \mathrm{~cm} \mathrm{GW}^{-1}$ and $\alpha=0$. The probe delay is fixed at $10 \mathrm{ps}$ to avoid transient effects. The pump focus position is shifted with respect to the probe focus by adjusting the pump mirror with a micrometer drive. The pump energy $E_{\text {pump }}$ is $10.8 \mu \mathrm{J}$ on a focus with $r_{\text {pump }}$ $=80 \mu \mathrm{m}$. The probe frequency $\nu_{\text {probe }} / c$ is $7692 \mathrm{~cm}^{-1}$, with a focus size of $r_{\text {probe }}=25 \mu \mathrm{m}$. The dashed line in the graph is drawn to indicate the width of the pump intensity distribution. The measured relative reflectivity shows a minimum at the center of the pump distribution, and decreases away from 


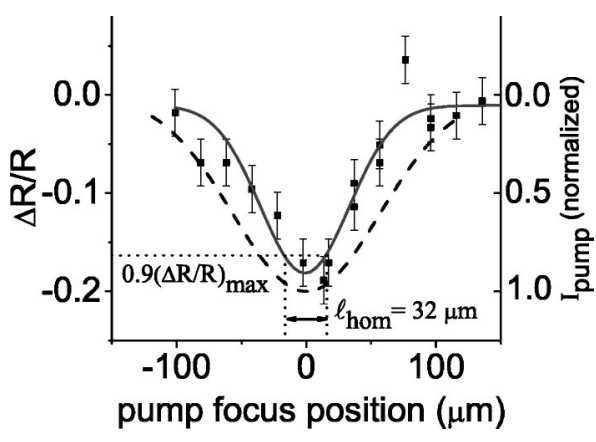

FIG. 7. Relative probe reflectivity measurement on a bulk Si sample for different lateral positions of the probe focus with respect to the probe focus. The probe delay is fixed at $10 \mathrm{ps}$. Pumping parameters: $\tau_{\text {pump }}=120 \mathrm{fs}$, $\nu_{\text {pump }} / c=6250 \mathrm{~cm}^{-1}, E_{\text {pump }}=10.8 \mu \mathrm{J}, r_{\text {pump }}=80 \mu \mathrm{m}, \tau_{\text {pump }}=120 \mathrm{fs}, \nu_{\text {probe }} / c$ $=7692 \mathrm{~cm}^{-1}, r_{\text {probe }}=25 \mu \mathrm{m}$. The squares are the measured data points, fitted with a Gaussian curve width of $68 \pm 10 \mu \mathrm{m}$ (solid curve). The horizontal dotted line indicates the level where the change in reflectivity has decreased by $10 \%$. From the vertical dotted lines, which indicate the intersections of the $90 \%$ line with the Gaussian fit of the data, we obtain a lateral homogeneity length of $32 \mu \mathrm{m}$, illustrating the excellent lateral homogeneity. The dashed curve indicates the measured width of the pump focus.

the center. The reflectivity data was fitted by a Gaussian curve (solid curve). The radius of this measured reflectivity minimum is $34 \pm 5 \mu \mathrm{m}$, which is considerably smaller than $r_{\text {pump. }}$ To obtain the lateral homogeneity length, we have determined the maximum lateral distance by the pump focus center for which $\Delta R / R$ remains within $10 \%$ of its maximum value (dotted lines). This distance turns out to be $16 \mu \mathrm{m}$ in both directions, corresponding to a homogeneity length of $32 \mu \mathrm{m}$. This illustrates that for a sample pumped by a pump beam with $r_{\text {pump }}=80 \mu \mathrm{m}$, the lateral homogeneity length is much better than $6 \mu \mathrm{m}$, which we required for switching of the density of states.

\section{SWITCHING HOMOGENEITY IN REAL PHOTONIC CRYSTALS}

The above analysis was done for bulk semiconductor samples. To obtain the homogeneity length in photonic crystals, the bulk absorption length is divided by the semiconductor filling fraction $\Phi$. In case of a $5 \%$ switch of the refractive index in a silicon photonic crystal with $\Phi=25 \%$ at $\nu_{\text {pump }} / c=5000 \mathrm{~cm}^{-1}$, the homogeneity length thus increases from 2.9 to $11.6 \mu \mathrm{m}$, which is twice the homogeneity requirement of $6 \mu \mathrm{m}$. For a higher pump frequency of $12500 \mathrm{~cm}^{-1}$, we find a homogeneity length of $2.3 \mu \mathrm{m}$, which is too low. This illustrates that the homogeneity required for switching of the density of states in silicon photonic crystals can only be performed at two-photon absorption frequencies.

In the analysis so far, the extinction of pump light due to random scattering inside the photonic crystals was neglected. We now discuss how to incorporate inevitable scattering in photonic samples. Scattering is quantified by the mean-free path $l_{\mathrm{mfp}}$ : the characteristic length over which a coherent beam becomes diffuse. The homogeneity length of light inside a photonic crystal is related to $l_{\mathrm{mfp}}$ and $l_{\mathrm{abs}}$ as

$$
l_{\text {hom }}=0.1 \times\left[\frac{1}{l_{\mathrm{abs}}}+\frac{1}{l_{\mathrm{mfp}}}\right]^{-1}
$$

In the limit of weak scattering, where $l_{\mathrm{mfp}} \gg l_{\mathrm{abs}}$, Eq. (10) reduces to $l_{\text {hom }}=0.1 \times l_{\mathrm{abs}}$. In the limit of strong scattering, where $l_{\text {mfp }} \ll l_{\text {abs }}$, the homogeneity length becomes $l_{\text {hom }}=0.1$ $\times l_{\mathrm{mfp}}$. As opposed to the adverse effect of scattering on the homogeneity in the $z$ direction, scattering will generally be favorable for lateral homogeneity, as pump light that is removed from the coherent pump beam is be scattered laterally.

Recently, our group has developed a quantitative model of the mean-free path inside photonic crystals. ${ }^{23}$ One of the main results of the analysis is that the mean-free path for pump light decreases with pump frequency squared $\omega_{\text {pump. }}^{-2}$. The absolute value of the mean-free path depends not only on frequency, but also on many properties of the crystal such as the unit cell size, the amount of disorder, and the refractive index contrast. As an example, we apply the model to a silicon inverse opal photonic crystal with a lattice parameter of $a=1240 \mathrm{~nm}$, corresponding to a bandgap frequency near $\omega_{\text {tele }}$. We assume combined size polydispersity and lattice displacements of $2 \%$ (which is beyond the current state of the art). From the model of Ref. 23, we obtain a mean-free path of 5.3 unit cells for pump light at $\nu_{\text {pump }} / c$ $=12500 \mathrm{~cm}^{-1}$. The corresponding homogeneity length, dominated by $l_{\mathrm{mfp}}$, would thus be 0.5 unit cells, well below our homogeneity requirement of 5 unit cells. For a 2.5 times lower pump frequency of $5000 \mathrm{~cm}^{-1}$, the model predicts a $(2.5)^{2}$ times larger mean-free path of $l_{\mathrm{mfp}}=33.3$ unit cells inside the crystal. Together with the earlier obtained absorption length of $l_{\mathrm{abs}}=116 \mu \mathrm{m}$ (=93 unit cells), we obtain a homogeneity length of $\left[(1 / 93+1 / 33)^{-1}\right] / 10=2.4$ unit cells. This result points out that even at pump frequencies near the two-photon absorption edge of silicon, the homogeneity requirement of 5 unit cells cannot be met in silicon inverse opals with a lattice parameter of $a=1240 \mathrm{~nm}$.

The scattering model predicts that decreasing the unit cell size will result in higher homogeneity, as this will reduce the relative pump frequency. Therefore, we consider silicon inverse opals with a reduced lattice parameter of $a$ $=900 \mathrm{~nm}$, corresponding to a bandgap near $8900 \mathrm{~cm}^{-1}$, just below the absorption edge of silicon. The scattering model predicts a larger mean-free path for pump light with a frequency of $5000 \mathrm{~cm}^{-1}$ of $l_{\mathrm{mfp}}=63$ unit cells. Using the absorption length of $116 \mu \mathrm{m}$ (=116 unit cells), we obtain a homogeneity length of $l_{\mathrm{hom}}=\left[(1 / 116+1 / 63)^{-1}\right] / 10=4.1$ unit cells, close to our homogeneity requirement.

Major improvements in switching homogeneity can be made by studying diamond-like structures, ${ }^{24,25}$ as the lattice parameter can be as low as $600 \mathrm{~nm}$ for a bandgap near $\omega_{\text {tele }}$ in such crystals. In such structures, the scattering model remains unchanged, apart from a constant prefactor that depends on the shape of the unit cell. We predict that reduced lattice parameters will decrease the relative pump frequency sufficiently to allow the homogeneous photonic density of states switching experiments.

\section{CONCLUSIONS}

We have discussed four important requirements for freecarrier-induced optical changes in semiconductors, with an 
emphasis on the density of states switching in photonic crystals: the amplitude of change in the refractive index, the time scale on which the switch takes place, the induced absorption, and the homogeneity of the induced change. We have demonstrated that the first two requirements can be met: a $10 \%$ change in $n^{\prime}$ in bulk silicon observed within 230 fs. We have also experimentally demonstrated sufficient lateral homogeneity in a bulk silicon. Furthermore, we have measured two-photon absorption coefficients in bulk silicon. The induced absorption was deduced to be low for carrier densities below $10^{20} \mathrm{~cm}^{-3}$.

We have discussed a nonlinear absorption model to describe the spatial homogeneity of optically generated electron-hole plasmas in semiconductors. We have introduced a homogeneity plot, which directly relates linear and two-photon absorption coefficients to the maximum homogeneity that can be achieved for any semiconductor. From such a plot, we conclude that for density of states switching in silicon photonic crystals, the optimum carrier density is about $10^{20} \mathrm{~cm}^{-3}$. To obtain the required homogeneity, the absorption coefficients must be minimized by a judicious choice of pump frequency. Due to peak intensity limitations the lowest pump frequency that can be chosen is around $5000 \mathrm{~cm}^{-1}$.

We have discussed the effect of scattering in photonic crystals on the pumping homogeneity. The homogeneity of switched photonic crystals turns out to be limited by scattering. We conclude that the homogeneity condition can barely be met in silicon inverse opals. We predict that in diamond structures the relative pump frequency will be small enough to allow homogeneous density of states switching experiments.

Finally, we have briefly discussed the ramifications for waveguides and modulators and we conclude that freecarrier switching is also useful for applications outside a photonic crystal.

\section{ACKNOWLEDGMENTS}

The authors wish to thank Irwan Setija and Rutger Voets (ASML) for deep UV lithography, Meint de Boer (TST) and Willem Tjerkstra for dry etching, and Leon Woldering for sample preparation. We also thank Allard Mosk and Ad Lagendijk for fruitful discussions and Martijn Wubs for deriving Eq. (7). This work is part of the research program of the
"Stichting voor Fundamenteel Onderzoek der Materie" (FOM), which is supported by the "Nederlandse Organisatie voor Wetenschappelijk Onderzoek" (NWO).

${ }^{1}$ Photonic Crystals and Light Localization in the 21st Century, edited by C. M. Soukoulis (Kluwer, Dordrecht, 2001).

${ }^{2}$ E. Yablonovitch, Phys. Rev. Lett. 58, 2059 (1987).

${ }^{3}$ S. John, Phys. Rev. Lett. 58, 2458 (1987).

${ }^{4}$ P. Lodahl, A. F. van Driel, I. Nikolaev, A. Irman, K. Overgaag, D. Vanmaekelbergh, and W. L. Vos, Nature (London) 430, 654 (2004).

${ }^{5}$ E. Yablonovitch, T. J. Gmitter, R. D. Meade, A. M. Rappe, K. D. Brommer, and J. D. Joannopoulos, Phys. Rev. Lett. 67, 3380 (1991).

${ }^{6}$ P. M. Johnson, A. F. Koenderink, and W. L. Vos, Phys. Rev. B 66, 081102 (2002).

${ }^{7}$ A. Liu, R. Jones, L. Liao, D. Samara-Rubio, D. Rubin, O. Cohen, R. Nicolaescu, and M. Paniccia, Nature (London) 427, 615 (2004).

${ }^{8}$ A. D. Bristow, J. P. R. Wells, W. H. Fan, A. M. Fox, M. S. Skolnick, D. M. Whittaker, A. Tahraoui, T. F. Krauss, and J. S. Roberts, Appl. Phys. Lett. 83, 851 (2003).

${ }^{9}$ A. Haché and M. Bourgeois, Appl. Phys. Lett. 77, 4089 (2000).

${ }^{10}$ S. W. Leonard, H. M. van Driel, J. Schilling, and R. B. Wehrspohn, Phys. Rev. B 66, 161102 (2002).

${ }^{11}$ V. R. Almeida, C. A. Barrios, R. R. Panepucci, M. Lipson, M. A. Foster, D. G. Ouzounov, and A. L. Gaeta, CLEO/IQEC and PhAST Technical Digest on CDROM (The Optical Society of America, Washington, DC, 2004), CTuFF3.

${ }^{12}$ K. Sokolowski-Tinten and D. von der Linde, Phys. Rev. B 61, 2643 (2000).

${ }^{13}$ D. A. Mazurenko, R. Kerst, J. I. Dijkhuis, A. V. Akimov, V. G. Golubev, D. A. Kurdyukov, A. B. Pevtsov, and A. V. Sel'Kin, Phys. Rev. Lett. 91, 213903 (2003).

${ }^{14}$ J. S. Kole, Ph.D. dissertation, Groningen, Rijksuniversiteit Groningen, 2003.

${ }^{15}$ For silicon, the value of $\tau_{\mathrm{D}}$ is $10^{-13}$, and the value for $m_{\mathrm{opt}}^{*}$ is 0.15 ; see Ref. 12.

${ }^{16}$ E. D. Palik, Handbook of Optical Constants of Solids (Academic, London, 1985)

${ }^{17}$ M. Dinu, F. Quochi, and H. Garcia, Appl. Phys. Lett. 82, 2954 (2003).

${ }^{18}$ J. F. Reintjes and J. C. McGroddy, Phys. Rev. Lett. 30, 901 (1973).

${ }^{19}$ A. J. Sabbah and D. M. Riffe, Phys. Rev. B 66, 165217 (2002).

${ }^{20}$ Our laser system provides high power pulses at two independently tunable frequencies. The setup consists of a regeneratively amplified Ti:Saf fs laser system (Spectra Physics Hurricane) that is used to pump two Topas optical parametric amplifiers (OPA). The OPA's have a continuously tunable output frequency between 3850 and $21050 \mathrm{~cm}^{-1}$ and produce $120 \mathrm{fs}$ pulses (for an autocorrelation graph see Fig. 2, lower panel) with a pulse energy $E_{\text {pulse }}$ of at least $20 \mu \mathrm{J}$ over the whole frequency range. Given a focal radius of $75 \mu \mathrm{m}$, the maximum intensity $I_{\max }$ is at least $1 \mathrm{TW} \mathrm{cm}^{-2}$.

${ }^{21}$ M. Sheik-Bahae, A. A. Said, T. H. Wei, D. J. Hagan, and E. W. van Stryland, IEEE J. Quantum Electron. 26, 760 (1991).

${ }^{22}$ A. Dargys and J. Kundrotas, Handbook on Physical Properties of Ge, Si, GaAs and InP (Science and Encyclopedia Publishers, Vilnius, 1994), and references therein.

${ }^{23}$ A. F. Koenderink and W. L. Vos, http://arxiv.org/abs/physics/0406052.

${ }^{24}$ K. M. Ho, C. T. Chan, and C. M. Soukoulis, Phys. Rev. Lett. 65, 3152 (1990).

${ }^{25}$ R. Hillebrand, St. Senz, W. Hergert, and U. Gösele, J. Appl. Phys. 94, 2758 (2003). 